\title{
Development of Environmental Specimen Holder for Hitachi S-5500 UHR SEM Microscope
}

\author{
S. Dogel ${ }^{*}$, D. Hoyle ${ }^{* *}$, M. Malac ${ }^{*}$, M. Salomons ${ }^{*}$, N. Mitsuhiro ${ }^{* *}$ and R. Wolkow ${ }^{*}$ \\ * National Institute for Nanotechnology, 11421 Saskatchewan Dr., Edmonton, Alberta, Canada, T6G \\ 2M9, ${ }^{* *}$ Hitachi High Technologies Canada, 89 Galaxy Blvd. Suite 14, Toronto, Ontario, Canada, \\ M9W 6A4
}

Environmental electron microscopy allows samples and processes to be studied in-situ in a gaseous environment. In many cases a sample can be examined in an essentially natural state. There are several options available to conduct in-situ investigations. One route is to use a dedicated environmental SEM or TEM instrument while another is to use a specially designed environment holder separating the high vacuum of the electron microscope from the sample by thin membranes $[1,2]$.

There are a considerable number of commercially available environmental holders which utilize electron-transparent membranes to encapsulate the volume containing the sample that is held at elevated pressure in gaseous environment. While such holders allow for high operating pressure, in some cases up to atmospheric pressure, they have limitations. At acceleration potentials typical for scanning electron microscopy the membranes can have a significant effect on the imaging performance or ability to acquire data due to scattering in the membrane. Moreover the membranes design prevents the acquisition of a secondary electron (SE) signal since the energy of the secondary electrons (below $100 \mathrm{eV}$ ) is too low to escape the membrane hence limiting signal collection to backscattered (BSE) and, in some cases, transmitted electrons. Although the electron energy can be significantly higher in a transmission electron microscope, the membrane design still contributes undesirable scattering reducing signal to background ratio.

Our design eliminates the membranes thus improving signal to background ratio compared to a typical environmental cell with membranes. It also allows us to use a standard SE detector to collect SE images. Our approach utilizes differential pumping around a windowless cell as shown in Figure 1. The combination of our environmental holder with unique capabilities of the Hitachi S-5500 ultrahigh resolution scanning electron microscope with cold field emission (CFE) gun [3] allows for collecting of a secondary electron image along with BSE and transmitted electrons in STEM mode of the instrument. Other advantages of the presented design are more obvious as the desired pressure of the experiment is decreased. For example at atmospheric pressure the scattering in membranes is a small contribution compared to the overall scattering in an environmental cell, while at lower pressure, of a few Pascals, the scattering in membranes is significant relative to overall interactions with the sample. Clearly, eliminating the membranes in experiments performed at moderate working pressure provides some advantage in signal to background ratio.

We have studied the utility and efficiency of our holder in charge reduction on the sample surface for imaging and analysis of non-conductive specimens. This is the basic and yet very common reason for introducing a gaseous environment around the specimen. An example of imaging a paper specimen at different gas pressures is shown in Figure 2 (see figure captions for details).

The environmental work in the S-5500 UHR SEM using our holder can potentially fit in between the operating regimes and information obtainable from large-chamber environmental SEM and an environmental TEM. The ability for the S-5500 UHR instrument to have such a large pressure difference over a short working distance allows for detection of the secondary electrons using the standard secondary electron detector while the system maintains the ultrahigh vacuum in the CFE gun area. 


\section{References}

[1] X. Zhang and T. Kamino, Microscopy Today, September 2006, p.16

[2] T. Kawasaki et al., Review of Scientific Instruments, 80 (2009) 113701

[3] http://www.hhtc.ca/microscopes/sem/s5500.htm

[4] Financial support of NINT/NRC, AITF and HHTC is gratefully acknowledged.

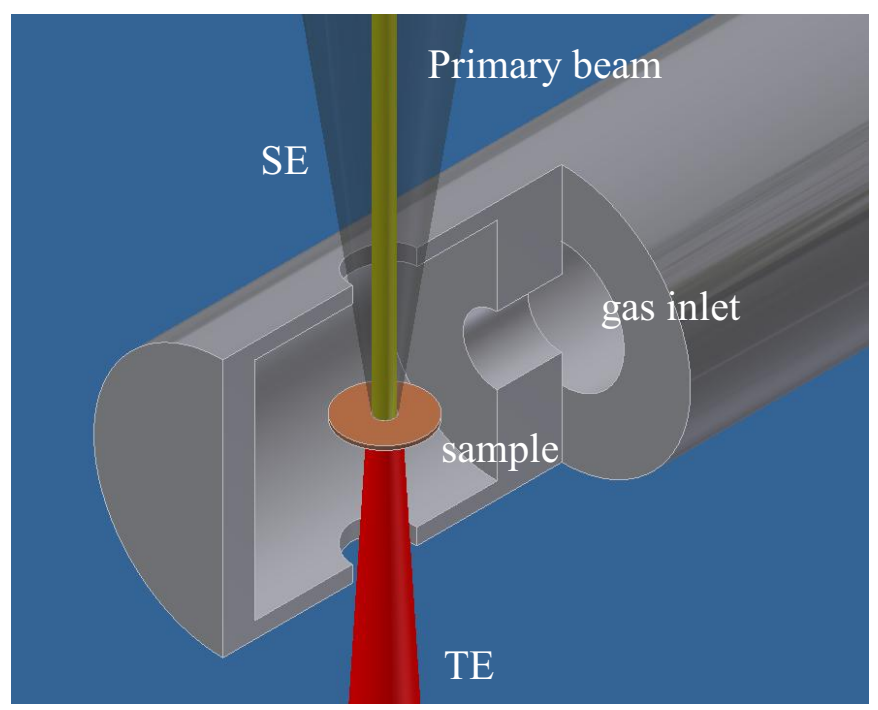

Figure 1. A schematic view of a windowless environmental holder: sample on TEM grid is positioned inside a small chamber which has one aperture above and one below the sample, and a gas delivery inlet.
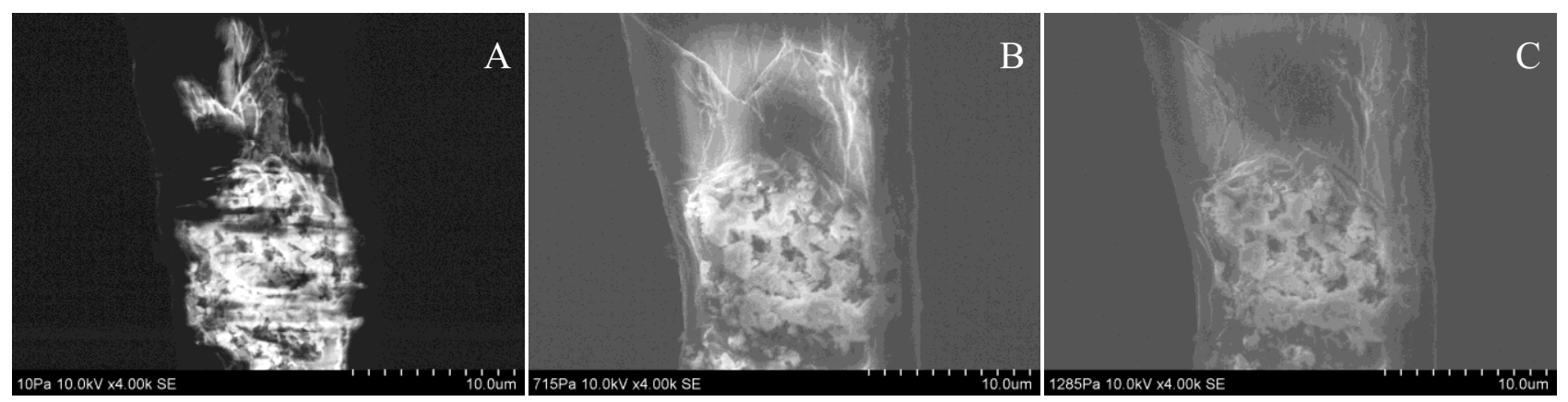

Figure 2. Paper specimen imaged in environmental holder in UHR S-5500 microscope at different pressures of nitrogen gas: a) about $10 \mathrm{~Pa}$ is insufficient to affect charging, b) approximately $700 \mathrm{~Pa}$, charging is largely eliminated while at about $1300 \mathrm{~Pa} \mathrm{c}$ ), charging effects appear to be suppressed further, but increased background from electron scattering in the working gas starts to becomes noticeable. For all images: SE, $10 \mathrm{kV}, \times 4.00 \mathrm{k}$, scale bar is $10 \mu \mathrm{m}$. 